\title{
DANDO VOZ A LAS MUJERES: REPRESENTACIONES SOCIALES Y \\ EXPERIENCIAS SOBRE LA LACTANCIA
}

\author{
GIVING VOICE TO WOMEN: SOCIAL REPRESENTATIONS AND EXPERIENCES
}

OF BREASTFEEDING

\author{
$M^{a}$ Dolores Pérez Bravo \\ Amparo Moreno Hernández \\ Universidad Autónoma de Madrid
}

\section{RESUMEN}

La lactancia materna, como una cuestión central en la investigación desde una perspectiva de género, constituye un proceso biopsicosocial, diverso y plural, que genera experiencias ambivalentes en las madres. Nuestra investigación tenía como objetivo comprender los pensamientos y experiencias de las madres sobre la lactancia. Hemos entrevistado individualmente a diez mujeres, madres primíparas y no primíparas, en dos momentos distintos. Los resultados muestran que las mujeres participantes tuvieron lactancias inferiores al tiempo recomendado por las organizaciones sanitarias, mencionaron razones relacionadas con el bienestar de sus hijos/as como principales motivos para amamantar y sus parejas aparecían como los principales apoyos sociales.

Palabras clave: lactancia materna, maternidad, género, feminismo.

\section{ABSTRACT}

Breastfeeding is a central issue in research from a gender perspective. We consider breastfeeding as a biopsychosocial, diverse and plural process, which generates ambivalent experiences in mothers. Our research was aimed to increase the understanding of thoughts and experiences of mothers. Ten women, primiparous and non-primiparous mother, were interviewed at two moments in time. The results showed that the breastfeeding period was shorter than the time recommended by health organizations, the reasons to breastfeed were related to the well-being of their children and women's social support came from their partners.

Keywords: Breastfeeding, Motherhood, Gender, Feminism. 


\section{Introducción}

Nuestro estudio sobre las representaciones de la lactancia se originó dentro de un proyecto más amplio que relacionaba las concepciones sobre la maternidad y la lactancia. Partimos de la idea de que ambas cuestiones deben abordarse desde una perspectiva de género. De hecho, una y otra han sido objeto de numerosas reflexiones desde el ámbito social, político, científico o filosófico y han dado cabida a diferentes planteamientos teóricos a lo largo de la historia. Cuatro son los núcleos fundamentales que hemos utilizado como organizadores de estas discusiones teóricas: la identificación entre identidad femenina y maternidad, la mitificación o idealización de la maternidad y los estereotipos de «buena» y «mala madre», la existencia o ausencia del instinto maternal y «amor materno» (Moreno, 2009) (Ver Tabla 1).

Tabla 1. Los debates sociales dicotómicos de la maternidad

\begin{tabular}{|l|l|}
\hline $\begin{array}{l}\text { Presencia de la maternidad en la identidad femenina: } \\
\text { Ser Mujer = Ser Madre }\end{array}$ & $\begin{array}{l}\text { Identidad femenina no vinculada a la maternidad: } \\
\text { Ser mujer =/= Ser madre }\end{array}$ \\
\hline $\begin{array}{l}\text { Maternidad idealizada: } \\
\text { El ldeal de madre } \\
\text { Felicidad plena }\end{array}$ & $\begin{array}{l}\text { Maternidad real: } \\
\text { Experiencias maternales reales } \\
\text { Ambivalencia de sentimientos }\end{array}$ \\
\hline $\begin{array}{l}\text { Maternidad natural: } \\
\text { Instinto materno y amor materno }\end{array}$ & $\begin{array}{l}\text { Maternidad cultural: } \\
\text { Construcción social de la maternidad }\end{array}$ \\
\hline $\begin{array}{l}\text { Maternidad dicotómica: Categorías de madre } \\
\text { Estereotipos de «Buena» y «Mala» madre }\end{array}$ & $\begin{array}{l}\text { Multiplicidad de maternidades: } \\
\text { Pluralidad y diversidad de madres }\end{array}$ \\
\hline
\end{tabular}

De igual forma, podemos analizar la lactancia materna tomando como referencia estos cuatro puntos (Ver Tabla 2).

Tabla 2. Los debates sociales de la lactancia materna: Posturas dicotómicas

\begin{tabular}{|l|l|}
\hline $\begin{array}{l}\text { Presencia de la lactancia materna en la identidad materna: } \\
\text { Amamantar = Ser madre = Ser mujer. }\end{array}$ & $\begin{array}{l}\text { Identidad materna no vinculada a la lactancia: Amamantar } \\
=/=\text { Ser madre =/= Ser mujer. }\end{array}$ \\
\hline $\begin{array}{l}\text { La lactancia materna en la representación de la «buena» y la «mala } \\
\text { madre». }\end{array}$ & $\begin{array}{l}\text { Pluralidad de lactancias: Diversidad de experiencias de } \\
\text { lactancia. }\end{array}$ \\
\hline La lactancia como instinto biológico. & La lactancia materna como construcción social. \\
\hline Idealización de la lactancia materna & Experiencias reales maternas con la lactancia. \\
\hline
\end{tabular}

Nuestra propuesta teórica entiende la lactancia materna y la maternidad como procesos biopsicosociales, diversos y plurales, que generan experiencias ambivalentes en las 
mujeres y cuya idealización social es digna de reflexión. Por ello, entendemos que hay que indagar, a través de investigaciones de género, sobre las opiniones femeninas acerca de estas cuestiones. Consideramos clave el «dar voz» a las mujeres para que cuenten abiertamente sus experiencias maternales y lactantes y de este modo aproximarnos a sus representaciones personales. Esperamos obtener, con esta metodología cualitativa, un conocimiento más profundo de la diversidad maternal, de sus demandas y necesidades como mujeres, madres lactantes o no lactantes.

Tal y como plantean Díaz, Catalán, Fernández y Granados (2011: 488) «son necesarias futuras investigaciones para lograr una visión amplia del fenómeno de las necesidades de las madres». Realizar investigaciones centradas en este aspecto nos ayudará a desarrollar líneas de trabajo en la promoción de la lactancia materna, que nos guíen en la articulación de un adecuado apoyo social, cultural, político, sanitario y familiar, al acercarnos más a la realidad vivida por las madres que amamantan, basándonos en sus propias circunstancias personales e idiosincráticas. Además, evitaremos la transmisión de una visión idílica de la maternidad y la lactancia y se contribuiría a no generar en las mujeres sentimientos de culpabilidad y/o ansiedad por no ajustarse a la demanda social y al ideal de «buena madre».

Hasta el momento, la mayoría de las investigaciones sobre lactancia materna, realizadas desde el campo sanitario, se han centrado en reforzar el papel beneficioso y saludable del amamantamiento obviando el papel de la mujer principalmente. Tal es así, que León-Cava, Lutter, Ros y Martín (2002) hicieron una extensa revisión bibliográfica con los artículos científicos que mostraban los beneficios de la lactancia materna. Por tanto, llevar también a cabo estudios con un enfoque de género para conocer cuáles son las vivencias y las experiencias de las mujeres con la lactancia materna, así como sus conocimientos, sus opiniones personales, sus creencias y sus actitudes se erige como una tarea prioritaria. En esta misma línea, consideramos imprescindible averiguar qué sentimientos y pensamientos les genera a las mujeres el amamantamiento o la incapacidad para lactar y cuáles son los beneficios, ventajas y los inconvenientes que creen que tiene la lactancia materna.

Resulta igualmente necesario analizar cuáles son las complicaciones y las dificultades, tanto a nivel social como personal, a las que éstas se enfrentan cuando optan por la decisión de dar el pecho a sus criaturas 0 , por el contrario, eligen la lactancia artificial, ya sea por decisión personal o por recomendaciones médicas en las que se desaconseja la lactancia materna tales como la galactosemia, la infección por VIH o HTLV, medicación materna que podría poner en riesgo al bebé, etc. 


\section{Objetivo de la investigación}

El estudio que hemos llevado a cabo va encaminado a examinar qué pensaban, sentían y opinaban las madres sobre la lactancia materna, con qué inconvenientes y dificultades personales y sociales se enfrentaban cuando tenían que dar el pecho a sus hijos o hijas, cómo valoraban esa conducta y a qué presiones sociales se habían visto sometidas en sus decisiones maternas. En definitiva, nuestra investigación se centraba en conocer el punto de vista de la mujer sobre varios aspectos de la lactancia materna e indagar en sus sentimientos y pensamientos al respecto.

\section{Aspectos metodológicos}

Desde un punto de vista metodológico, la investigación que presentamos es un estudio fenomenológico, con un diseño descriptivo y una metodología cualitativa. Confeccionamos una entrevista semiestructurada, con preguntas abiertas sobre tres grandes bloques temáticos (Datos sociodemográficos, maternidad, lactancia materna). En concreto, nuestro guión de entrevista sobre la lactancia incluía preguntas relacionadas con la duración de la lactancia, las razones para dar el pecho, los beneficios y los inconvenientes, etc. (Ver Tabla 3).

Se entrevistó a un grupo de diez mujeres con diferentes características individuales. Cinco de las mujeres participantes eran madres primíparas y las otras cinco ya tenían un hijo/a previamente y podían tener o no tener experiencia previa con el amamantamiento. Una aportación significativa de nuestra investigación reside en que las entrevistamos en dos momentos distintos: una primera entrevista fue realizada en el tercer trimestre de embarazo y una segunda entrevista a los $4-5$ meses del parto (ver Tabla 4). Una vez realizadas y grabadas en voz, las entrevistas se transcribieron literalmente. Posteriormente, con la ayuda del programa informático de análisis cualitativo Atlas.Ti (Versión 6.0), se codificaron las citas textuales con mayor interés para el estudio y el posterior análisis e interpretación de los resultados, intentando identificar algunas diferencias entre los dos momentos de la entrevista y entre las madres primíparas y no primíparas, sobre todo, con respecto a las representaciones sociales de las madres en relación con la maternidad y la lactancia materna. Entendiendo que estas representaciones, aunque residan en la persona, suponen un conocimiento socialmente elaborado y compartido, que ha surgido de las prácticas que la persona lleva a cabo en contextos de relación y, por tanto, están influidas por las condiciones sociales en que se desarrollan las personas. Como afirma Delval (2011), las personas tenemos la capacidad de elaborar representaciones sobre la realidad que nos permiten actuar sobre 
la misma y anticipar lo que puede suceder. En concreto, Ibáñez (1988: 55) plantea que las representaciones sociales sirven para que las personas describan, clasifiquen y expliquen los fenómenos de las realidades cotidianas y producen los significados que necesitan para comprender, actuar y orientarse en su medio social.

Tabla 3. Preguntas de investigación sobre el tema de la Lactancia Materna

\begin{tabular}{|c|c|}
\hline LACTANCIA MATERNA & PREGUNTAS DE INVESTIGACIÓN \\
\hline RAZONES DE DAR EL PECHO & $\begin{array}{l}\text { 1. ¿Cuáles son las razones de dar el pecho de las madres primíparas y las madres con } \\
\text { hijos/as previos/as? } \\
\text { 2. ¿Existe algún tipo de relación entre las razones de dar pecho y los beneficios de } \\
\text { amamantar? }\end{array}$ \\
\hline DURACIÓN DE LA LM & $\begin{array}{l}\text { 1. ¿Cuáles son las expectativas de duración de LM que tienen las madres y las razones } \\
\text { de dar el pecho hasta esa edad de sus criaturas? } \\
\text { 2. ¿Cuál es la duración media de la LM que dan las madres del estudio y las razones } \\
\text { de haber destetado/abandonado la lactancia a esa edad? }\end{array}$ \\
\hline FUENTES DE INFORMACIÓN & $\begin{array}{l}\text { 1. ¿Cuáles son las fuentes de información sobre la LM que tienen las madres primeri- } \\
\text { zas y las madres con hijos/as previos/as? }\end{array}$ \\
\hline $\begin{array}{l}\text { BENEFICIOS Y VENTAJAS DE LA } \\
\text { LACTANCIA MATERNA }\end{array}$ & $\begin{array}{l}\text { 1. ¿Cuáles son los beneficios y las ventajas de la LM que manifiestan las madres primí- } \\
\text { paras y con hijos/as previos/as? } \\
\text { 2. ¿Existe alguna relación entre los beneficios y las razones de dar teta o con tener una } \\
\text { visión idealizada de la lactancia? }\end{array}$ \\
\hline $\begin{array}{l}\text { INCONVENIENTES Y DIFICULTA- } \\
\text { DES DE LA LM }\end{array}$ & $\begin{array}{l}\text { 1. ¿Cuáles son los inconvenientes y las dificultades de la LM que manifiestan las ma- } \\
\text { dres primerizas y con hijos/as previos/as? } \\
\text { 2. ¿Existe alguna relación entre los inconvenientes y las razones de abandonar o } \\
\text { finalizar la lactancia? }\end{array}$ \\
\hline $\begin{array}{l}\text { APOYOS SOCIALES Y TOMA DE } \\
\text { DECISIONES }\end{array}$ & $\begin{array}{l}\text { 1. ¿Cuáles son los apoyos con los que cuentan las madres en sus decisiones de dar } \\
\text { pecho/biberón y en sus vivencias con la } L M \text { ? } \\
\text { 2. ¿Las madres sufren o sienten algún tipo de presión social a la hora de decidir dar el } \\
\text { pecho a sus criaturas? } \\
\text { 3. ¿Cómo toman las decisiones sobre lactar o no lactar las madres primerizas y con } \\
\text { hijos/as previos/as? }\end{array}$ \\
\hline $\begin{array}{l}\text { REPRESENTACIONES SOCIALES } \\
\text { DE LA LM }\end{array}$ & $\begin{array}{l}\text { 1. ¿Qué opinan las madres sobre la lactancia prolongada, el amamantamiento en } \\
\text { público, la recomendación de la OMS? } \\
\text { 2. ¿Qué opiniones creen que hay en la sociedad sobre dichos temas? }\end{array}$ \\
\hline $\begin{array}{l}\text { VIVENCIAS PERSONALES CON } \\
\text { LA LM }\end{array}$ & $\begin{array}{l}\text { 1. ¿Qué manifiestan las madres sobre sus vivencias y experiencias personales con la } \\
\text { lactancia materna? } \\
\text { 2. ¿ } \text { Hay diferencias entre sus expectativas previas y la experiencia que tienen? }\end{array}$ \\
\hline $\begin{array}{l}\text { SENTIMIENTOS POSITIVOS vs } \\
\text { NEGATIVOS HACIA LA LM }\end{array}$ & $\begin{array}{l}\text { 1. ¿Cuáles son los sentimientos positivos y negativos hacia la } L M \text { que manifiestan las } \\
\text { madres primíparas y con hijos/as previos/as sobre los sentimientos positivos y negati- } \\
\text { vos hacia la LM? } \\
\text { 2. ¿Cuáles son los sentimientos que les genera a las madres la incapacidad de dar teta } \\
\text { o el abandono de la lactancia materna? }\end{array}$ \\
\hline
\end{tabular}


Tabla 4. Número de entrevistas realizadas

\begin{tabular}{|c|c|c|}
\hline $\begin{array}{c}\mathbf{1}^{\mathbf{a}} \text { entrevista } \\
\text { (Tercer trimestre de gestación) }\end{array}$ & $\begin{array}{c}\mathbf{2}^{\mathbf{a}} \text { Entrevista } \\
(\mathbf{4}-\mathbf{5} \text { meses posparto) }\end{array}$ & Total de Entrevistas \\
\hline $\begin{array}{c}\text { Mujeres embarazadas primíparas } \\
(\mathbf{N}=\mathbf{5})\end{array}$ & $\begin{array}{c}\text { Madres primíparas } \\
(\mathrm{N}=5)\end{array}$ & $\mathbf{N}=\mathbf{1 0}$ \\
\hline $\begin{array}{c}\text { Madres embarazadas con hijos/as } \\
(\mathbf{N}=\mathbf{5})\end{array}$ & $\begin{array}{c}\text { Madres con hijos/as } \\
(\mathrm{N}=5)\end{array}$ & $\mathbf{N}=\mathbf{1 0}$ \\
\hline $\mathbf{N}=\mathbf{1 0}$ & $\mathbf{N}=\mathbf{1 0}$ & $\mathbf{N}=\mathbf{2 0}$ \\
\hline
\end{tabular}

\section{Resultados}

Desde el marco general de las relaciones entre lactancia y maternidad, los resultados mostraron que las participantes entendían la maternidad como una parte fundamental de la identidad femenina y mantenían una visión generalmente estereotipada de este proceso. En mayor medida, las madres primíparas expresaban que no concebían la vida sin hijos/as y que es una experiencia por la que las mujeres deberían pasar, por ejemplo, R.G.O. (44 años, primípara, $1^{a}$ entrevista) dijo textualmente: «algo muy grande que todas las mujeres tenían que experimentar» y C.P.M. (38 años, con hijos/as previos/as, $1^{a}$ entrevista) declaró: «algo como que una mujer tiene que pasar porque yo creo que si no lo pasas, no puedes saber qué es». Las madres participantes hablaban también de la maternidad como un proceso de cambio vital que conlleva una gran responsabilidad y expresaron sentimientos ambivalentes en torno a la maternidad y la lactancia. De hecho, mencionaban sentimientos tanto positivos como negativos, siendo algunos negativos comunes tales como el miedo, la culpabilidad - la preocupación. Para nosotras, este dato es crucial ya que resulta fundamental respetar la expresión emocional ambivalente de las madres, para ayudar a mostrar socialmente la verdadera realidad de la maternidad y la lactancia.

La lactancia materna no apareció espontáneamente al referirse a la maternidad, al igual que en el estudio de Castilla (2005). Tan sólo cuando se les preguntaba de forma explícita, la mayoría verbalizaba que el amamantamiento no revaloriza a las madres aunque creían que las madres que dan teta parecen ser mejor consideradas socialmente. Tanto es así que algunas de las participantes narraban cómo se habían sentido juzgadas socialmente como «malas madres» cuando abandonaron precozmente la lactancia materna y reflejaban haber experimentado cierta presión social, sobre todo desde el ámbito sanitario, para continuar amamantando a sus hijos/as. Así M.A.S. (26 años, primípara, $1^{a}$ entrevista) verbalizó «creo que en la sociedad está visto pues como que si no le das el pecho a tu hijo, pues no eres buena madre. Pero yo a nivel mío, personal, pienso

112

Dossiers Feministes, 22, 2017, 107-117 - ISSN: $1139-1219$ - DOI: http://dx.doi.org/10.6035/Dossiers.2017.22.7 
que no eres mejor madre». Y, C.P.M. (38 años, con hijos/as previos/as, $2^{a}$ entrevista) manifestó: «te crean un sentimiento de culpabilidad... de decir: [...] ¡que no es que yo no se lo quiera dar!, ique es que no me sale! [...] Llega un momento en que es una frustración, es un sentimiento de....bueno es que rompes a llorar diciendo que es que pareces que eres una mala madre».

En cuanto a los resultados obtenidos sobre los motivos para amamantar algunos coinciden con estudios previos (Llorens et. al, 2007; Marcos y Zapata, 1998; Martín-Pérdiz, 2008; Martínez, 2010). En nuestro estudio, las madres participantes han argumentado diferentes razones para amamantar centradas fundamentalmente en los beneficios que la lactancia materna tiene sobre el bebé, obviando prácticamente sus propias necesidades. La mayoría aluden a cuestiones nutricionales considerando la leche materna como el mejor alimento para la criatura, a cuestiones saludables y beneficiosas para el bebé, y a la creación del vínculo afectivo entre madre-hijo/a. Desde nuestro punto de vista, creemos que esto se deriva de la representación que tienen de la buena madre como aquella que atiende las necesidades de sus hijos/as y se preocupa por su bienestar. Por lo tanto, basándonos en esta representación, sus razones para lactar deberían ir enfocadas a satisfacer las necesidades de sus criaturas, pues hemos comprobado a través de sus discursos cómo eran conocedoras de los innumerables beneficios que tiene la lactancia materna (y que son claramente defendidos desde el terreno sanitario). En definitiva, podríamos plantear que la promoción social y sanitaria de la lactancia materna centrada únicamente en los beneficios que tiene para la criatura, parece impedir que las madres manifiesten argumentos personales para lactar centrándose en ellas mismas, sus necesidades o beneficios propios y refuerza que sean argumentos enfocados principalmente en el bienestar del propio hijo/a. Tanto es así que solo dos de todas ellas son las que hacen referencia al beneficio propio, expresando «también, por el beneficio mío [...]. Es muy bueno para la madre en el sentido de la recuperación» (C.M.S., 29 años, $1^{a}$ entrevista) y «dicen que para el pecho es bueno también, que reduce el cáncer de mama, que te ayuda a coger el peso pronto» (N.E.V., 38 años, $1^{a}$ entrevista).

Creemos, pues, que sería importante conseguir que las madres también fueran capaces de argumentar en mayor medida razones personales para amamantar; de decidir libremente no dar el pecho o de abandonar la lactancia cuando les resulta imposible, insoportable o insufrible, sin sentirse juzgadas, presionadas o menos valoradas en su papel materno. En ese sentido, compartimos la idea de González sobre que

Las madres no pueden convertirse en rehenes o víctimas de la lactancia materna. El bebé es muy importante, pero también lo es la madre y apenas se tiene en cuenta sus necesidades como persona y como mujer. A veces, una lactancia artificial satisfactoria y placentera es mejor que una mala lactancia materna (González, 2015: 36). 
Por otro lado, hemos encontrado que las madres participantes calificaban sus vivencias maternales como satisfactorias aunque hubieran tenido experiencias complicadas con la lactancia y muchas de sus expectativas previas con la misma no se cumplieran. En sus experiencias lactantes, han identificado algunos inconvenientes (dolores físicos, hipogalactia) de la lactancia materna. Algunas de ellas planteaban que no habían recibido información suficiente sobre las complicaciones que pueden surgir durante la lactancia y la manera de superarlas. Por ejemplo, M.A.S. (26 años, primípara, $2^{a}$ entrevista) planteaba «también debían informar que sí te pasan estas cosas [...] pienso que deberían hacer más hincapié en la madre, decir: ¡mira, pues os puede pasar este tipo de cosas! [...] debían informar así en los cursillos y las clases que dan, tanto lo positivo que tiene, que lo tiene...si yo las sigo viendo a pesar de la experiencia mala que he tenido». Además, consideraban que la reincorporación al mundo laboral, la conciliación entre la vida familiar y personal, las repercusiones en la trayectoria profesional son dificultades, inconvenientes $y / o$ aspectos negativos a tener en cuenta tanto de la lactancia materna como de la maternidad. Tal es así que M.A.S. (26 años, primípara, $1^{a}$ entrevista) declaraba «me preocupa, pues eso, a la hora de irme a trabajar [...] el sentimiento de culpa cuando lo tenga que dejar para ir a trabajar y tenga que dejarle [...] eso es lo más negativo que veo: el día que me tenga que incorporar, pues supongo que será un valle de lágrimas». R.L.M. (32 años, primípara, $1^{a}$ entrevista) decía «el mayor impedimento que existe para la lactancia materna, para una mujer que quiere realmente [...] dar leche es sobre todo laboral».

Ante este resultado, nos gustaría poner de manifiesto la necesidad de formar e informar adecuadamente a las mujeres desde el ámbito sanitario. Es preciso instruir y apoyar a las madres en las dificultades, inconvenientes y complicaciones que pueden producirse en el establecimiento y proceso de la lactancia, así como en la manera de superarlas. No resulta suficiente ofrecerles información sobre las ventajas del amamantamiento e insistirles en que lacten, sino que deberíamos contarles las complicaciones que pueden surgir, los obstáculos a los que podrían enfrentarse y las posibles soluciones. Es inevitable tener que aportarles recursos personales y lugares a los que acudir para recibir el mejor apoyo emocional y asesoramiento profesional. Observamos, pues, la necesidad de un seguimiento sanitario más respetuoso, más individualizado y personalizado en función de las características y circunstancias personales de las madres. De la misma forma, que a nivel más general, abogamos por una revisión de las actuales políticas sociales y laborales para establecer medidas más adecuadas de apoyo a la maternidad y lactancia.

Por último, al igual que en otros estudios (Belintxon et. al, 2011), hemos encontrado que los principales apoyos para las madres participantes en la toma de decisiones son sus parejas. En nuestro estudio, también, nombraban a otras mujeres cercanas (madres, tías,

114

Dossiers Feministes, 22, 2017, 107-117 - ISSN: $1139-1219$ - DOI: http://dx.doi.org/10.6035/Dossiers.2017.22.7 
abuelas, grupos de apoyo) de las que decían haber obtenido alguna información concreta sobre la lactancia. El apoyo del padre solo aparece reflejado en nuestra investigación cuando las madres participantes tenían que tomar la decisión de abandonar la lactancia por resultarles muy dolorosa físicamente. Sin embargo, el apoyo del padre no se ha visto reflejado en otros aspectos planteados durante la entrevista. Este hecho hace de nuevo patente que la responsabilidad del cuidado de los hijos/as sigue recayendo en gran medida en las mujeres. Por el contrario, entendemos que el padre desempeña un papel crucial en la lactancia y la maternidad, que incluye acompañar emocionalmente a las madres y corresponsabilizarse en la crianza y cuidado de los hijos/as y de todas las tareas domésticas. Olza (2013: 24) plantea que, cuando se habla de lactancia, «a los padres casi nadie les pregunta qué opinan ni cómo llevan ellos la lactancia, y tampoco se les suele informar de cómo ayudar a sus compañeras durante la lactancia». Ante este resultado, nos hemos planteado que sería muy interesante conocer también cuáles son las creencias y representaciones masculinas en torno a la lactancia materna, la maternidad y la paternidad en futuros proyectos de investigación.

\section{Conclusiones}

En conclusión, los resultados hallados refuerzan nuestro planteamiento teórico sobre la lactancia materna como un proceso que entremezcla lo biológico, lo psicológico y lo social, que se muestra muy heterogéneo y variado, que origina experiencias ambivalentes en las mujeres y cuya idealización merece una reflexión profunda en nuestra sociedad actual. A las autoras nos queda pendiente, en un futuro, ampliar y mejorar nuestro proyecto en diferentes direcciones. Pretendemos acceder a una muestra mayor, incorporar nuevos métodos de recogida de información (por ejemplo, a través de grupos de discusión, blogs de madres, observación en grupos de apoyo a la lactancia,...) o tener en cuenta otras variables importantes en la selección de las mujeres (por ejemplo, mujeres lesbianas, mujeres que hayan acudido a grupos de apoyo a la lactancia, madres acogedoras o adoptivas, ...). En definitiva, intentaremos seguir ampliando la muestra de mujeres (y por qué no, también de hombres) para continuar profundizando en esta diversidad de la realidad femenina y conseguir un mayor conocimiento teórico siempre desde la propia voz de las mujeres. 


\section{Referencias bibliográficas}

Belintxon-Martín, Maider, Zaragüeta, Maria, Adrián, M.C. y López-DiCastillo, Olga (201 1): «El comienzo de la lactancia: experiencias de madres primerizas». Anales del Sistema Sanitario de Navarra. № 34 Vol. 3, pp. $409-418$.

CAstilla, M $M^{a}$ Victoria (2005): «La ausencia del amamantamiento en la construcción de la buena maternidad». Revista de estudios de Género La Ventana. №22, pp. 188-218.

Delval, Juan (2011): El mono inmaduro. El desarrollo psicológico humano, Madrid, Los Libros de La Catarata.

Díaz, Jorge, Catalán, Daniel, Fernández, Milagros y Granados, Genoveva (2011): «La comunicación y la satisfacción de las primíparas en un servicio público de salud». Gaceta Sanitaria. № 25 Vol. 6, pp. 483-489.

GonzÁleZ, Jose María (2015): Víctimas de la lactancia materna. ¡Ni dogmatismos ni trincheras!. Madrid, Akane Ediciones.

IBÁÑEZ, Tomás (1988): Ideologías de la Vida Cotidiana, Barcelona, Editorial Sendai.

León-CAVA, Natalia, LutTer, Chessa, Ross, Jay y Martín, Luann (2002): Cuantificación de los beneficios de la Lactancia Materna: Reseña de la Evidencia. Buenos Aires, Programa de alimentación y nutrición (HPN) y Organización Panamericana de la Salud (OPS).

Llorens, Beatriz, Ferrer, Elena, Morales, Vanesa, y Alenda, Ana (2007): «Creímos en la lactancia materna. Una aproximación de la experiencia en tres generaciones». Index de Enfermería, № 16 Vol. 58.

MARCOS, E. y ZAPATA, Rosa (1998): «Actitudes de algunas madres respecto a la lactancia materna». Enfermería Científica. № 194-195, pp. 56-59.

Martínez, Juan Miguel (2010): «Factores asociados al abandono de la lactancia materna». Metas de Enfermería. № 13 Vol. 6, pp. 61-67.

Martín-Perdíz, Antonia (2008): «Entre la idealización de la lactancia materna y la realidad. La experiencia de amamantar a un recién nacido prematuro». Enfermería Clínica. $\mathrm{N}^{\circ}$ 18 Vol. 5, pp. 279-280.

MORENO, Amparo (2009). Los debates sociales de la maternidad: maternidad y maternidades. En C. Bernis, R. López, y P. Montero (Eds.), Determinantes biológicos, psicológicos y sociales de la maternidad en el Siglo XXI: Mitos y realidades, Madrid, Universidad Autónoma de Madrid, pp. 3-19.

OlzA, Ibone (2013): Lactivista, Tenerife, Ed. Ob Stare.

PÉREZ, Ma Dolores (2015). Maternidades y lactancias. La lactancia materna desde la perspectiva de género (Tesis doctoral no publicada), Madrid, Universidad Autónoma de Madrid.

116

Dossiers Feministes, 22, 2017, 107-117 - ISSN: $1139-1219$ - DOI: http://dx.doi.org/10.6035/Dossiers.2017.22.7 
ROMERO, Ma Elena y JIMENO, Jose Francisco (2006): «La lactancia materna en el niño mayor». Medicina Naturista. № 10, pp. 646-652.

VÁsQUEZ, Mirith, ROMERO, Ángela y RIVAS, Aneth (2012): «Motivaciones de la madre para amamantar: una experiencia a partir de la capacidad de escuchar». Revista de la Facultad de Ciencias de la Salud de Universidad de Magdalena. № 9 Vol. 2, pp. 116-122.

Recibido el 10 de marzo de 2017 Aceptado el 6 de junio de 2017 BIBLID [1 139-1219 (2017) 22: 107-117] 